\title{
EFEMP1 rare variants cause juvenile-onset open angle glaucoma in families from the Philippines
}

\author{
Edward Collantes ${ }^{1}$, Manuel Delfin ${ }^{2}$, Bao Jian Fan ${ }^{1}$, Justine Torregosa ${ }^{3}$, Christine \\ Siguan-Bell $^{3}$, Nilo Florcruz ${ }^{4}$, Jose Martinez ${ }^{5}$, Barbara Masna-Hidalgo ${ }^{6}$, Vincent Guzman ${ }^{6}$, \\ Jewel Anotado-Flores ${ }^{6}$, Faye Levina ${ }^{7}$, Sophia Hernandez ${ }^{8}$, Anthony Collantes ${ }^{9}$, Michael \\ Sibulo $^{10}$, Shisong Rong ${ }^{1}$, and Janey Wiggs ${ }^{1}$ \\ ${ }^{1}$ Harvard Medical School \\ ${ }^{2}$ Manila Doctors Hospital \\ ${ }^{3}$ Cebu Institute of Medicine \\ ${ }^{4}$ University of the Philippines Manila \\ ${ }^{5}$ East Avenue Medical Center \\ ${ }^{6}$ Western Visayas Medical Center \\ ${ }^{7}$ Jose R Reyes Memorial Medical Center \\ ${ }^{8}$ Umeå Universitet Medicinska fakulteten \\ ${ }^{9}$ Our Lady of Fatima University College of Medicine \\ ${ }^{10}$ University of Santo Tomas Faculty of Medicine and Surgery
}

July 19, 2021

\begin{abstract}
Juvenile open angle glaucoma (JOAG) is a severe type of glaucoma with childhood onset and dominant inheritance. Using exome sequencing we identified 3 independent families from the Philippines with rare EFEMP1 variants (c.238A>T, p.Asn80Tyr; c.1480T >C p.Ter494Glnext*29; and c.1429C > T, p.Arg477Cysteine ) co-segregating with disease. Affected variant carriers (N= 34) exhibited severe disease with average age of onset of 16 years and with $76 \%$ developing blindness. To investigate functional effects, we transfected COS7 cells with vectors expressing the three novel EFEMP1 variants and showed that all three variants found in JOAG patients caused significant intracellular protein aggregation and retention compared to wild type and also compared to EFEMP1 variants associated with other ocular phenotypes including an early-onset form of macular degeneration, Malattia Leventinese/Doyne's Honeycomb retinal dystrophy. These results suggest that rare EFEMP1 coding variants can cause JOAG through a mechanism involving protein aggregation and retention, and that the extent of intracellular retention correlates with disease phenotype. This is the first report of EFEMP1 variants causing JOAG, expanding the EFEMP1 disease spectrum. Our results suggest that EFEMP1 mutations appear to be a relatively common cause of JOAG in Filipino families, an ethnically diverse population.
\end{abstract}

\section{Introduction}

Glaucoma is one of the most heritable of all human diseases (Wang et al., 2017) and exhibits both Mendelian (childhood onset) and complex (adult onset) inheritance (Wiggs and Pasquale, 2017). Affected patients typically develop elevated intraocular pressure (IOP) that causes irreversible degeneration of retinal ganglion cells and the optic nerve (Weinreb et al., 2016). Increased IOP in most patients is caused by impaired drainage of intraocular fluid from the eye through the trabecular meshwork and outflow pathways (Weinreb et al., 2014). 
Patients with childhood-onset glaucoma, defined as diagnosis before age 40, are affected by severe disease characterized by very high IOP, and without treatment are likely to become blind over their lifetimes (Papadopoulos et al., 2020). Juvenile-onset primary open-angle glaucoma (JOAG) is one type of childhood glaucoma that typically exhibits dominant inheritance. MYOC mutations account for disease in 15-20\% of JOAG families primarily with European Caucasian ancestry and $M Y O C$ does not appear to be a major cause of disease in other ethnic groups (Liu et al., 2021). To investigate the genetic etiology of JOAG in an ethnically diverse population, we identified and recruited 14 JOAG pedigrees from regions throughout the Philippines.

EFEMP1 (EGF-containing fibulin-like extracellular matrix protein 1) also known as Fibulin 3 is a member of the Fibulin family of extracellular matrix proteins characterized by tandem arrays of EGF (epidermal growth factor)-like domains and a C-terminal fibulin-type module (Figure 1B) (Kobayashi et al., 2007). Among the fibulin family members,EFEMP1 has higher ocular expression (Wagner et al., 2013) and is known to contribute to other ocular phenotypes. Common SNPs nearEFEMP1 on chromosome 2p16 have been associated with adult-onset glaucoma (POAG) (Gharahkhani et al., 2021) as well as IOP (Khawaja et al., 2018) and cup-to-disc ratio (CDR) an optic nerve quantitative trait related to POAG risk (Springelkamp et al., 2015). A low frequencyEFEMP1 variant p.Arg140Trp, present in 2 individuals in gnomAD has been identified in 5 affected individuals from an adult-onset POAG African-American family (Mackay et al., 2015) and a stop loss variant (Ter494Gluext*29) has been reported in 3 affected members of a Chinese POAG family (Liu et al., 2020). A single EFEMP1 missense allele (p.Arg345Trp) is known to cause Malattia Leventinese (MLVT) (also known as Doyne's Honeycomb dystrophy (DHRD; MIM:126600), a rare autosomal dominant retinal degeneration characterized by extracellular deposits (drusen) beneath the retinal pigment epithelium (RPE) (Stone et al., 1999). Among the many patients affected by MLVT/DHRD, only p.Arg345Trp has been identified as a causative mutation. Recently it has been suggested that the Arg345 residue is a mutation hot spot and that founder effects are not likely to account for the predominance of this mutation in MLVT/DHRD patients (Vaclavik et al., 2020). Notably, wildtype EFEMP1 is a secreted protein, while the p.Arg345Trp mutations leads to protein misfolding and reduced secretion (Marmorstein et al., 2002).

Here, we report three independent families from the Philippines with novel EFEMP1 rare variants segregating with JOAG. Functional studies suggest that the disease-associated EFEMP1 variants cause protein misfolding and intracellular aggregation and retention. These results expand the spectrum of EFEMP1 associated disease to include childhood glaucoma.

\section{Methods}

Ethical approval for this study was obtained from the Institutional Review Board of the Manila Doctors Hospital and Cebu Velez General Hospital, Philippines and the Mass General Brigham (MGB) IRB. In accordance with the tenets of the Declaration of Helsinki, written informed consent was obtained from the study subjects after explanation of the nature and possible consequences of the study.

\section{Study recruitment}

Subjects with juvenile open angle glaucoma (JOAG) were recruited from ophthalmology clinics. When possible, subjects underwent an ophthalmologic exam, which included visual acuity assessment, slit-lamp examination to evaluate the anterior segment, dilated fundus examination to document the structural changes of glaucoma, and gonioscopic examination to evaluate the angle structure. JOAG was defined as: 1) absence of secondary causes of glaucoma; 2) IOP $>21 \mathrm{mmHg}$; 3) open anterior chamber angle on gonioscopy (grade 3 or 4 of modified Shaeffer classification); 4) characteristic optic disc damage and/or typical visual field loss; and 5) IOP $>21 \mathrm{mmHg}$ in at least one eye in any family member aged between 3 and 40 years old. Pedigree information and disease status were ascertained from probands and family members. Asymptomatic family members were also invited to participate in the study and undergo a clinical examination and blood draw. Venipuncture was done on each subject and $10 \mathrm{cc}$ of venous whole blood was obtained. Genomic DNA was extracted from the blood samples with the DNeasy Blood DNA extraction kit (Qiagen, Valencia, CA) according to the manufacturer's instructions. 


\section{Exome sequencing}

Whole exome sequencing (WES) was performed at the Massachusetts Eye and Ear Ocular Genomics Institute using Agilent SureSelect Human All Exon v6 with the Illumina HiSeq 2000, or at the Broad Institute of MIT and Harvard using a TWIST Biosciences custom exome bait with the Illumina Novaseq 6000. We used the Ocular Genomics Institute bioinformatics pipeline (Consugar et al., 2015) for alignment and variant calling that includes the Burrows-Wheeler aligner (Li et al., 2010), GATK (https://gatk.broadinstitute.org/), GERP (http://mendel.stanford.edu/SidowLab/downloads/gerp/), Exome Aggregation Consortium (ExAC) (http://exac.broadinstitute.org), SIFT (http://sift.jcvi.org/), and PolyPhen-2 (http://genetics.bwh.harvard.edu/pph2/). A coverage depth cutoff of 10x was applied. Heterozygous was defined as a fraction of a variant base between $0.25-0.75$ and homozygous was defined as above 0.75 . Average coverage was $105 x$ for $99 \%$ of coding sequences.

\section{Variant filtering}

Ten genes (MYOC, CYP1B1, LTBP2, PITX2, FOXC1, PAX6, TEK, ANGPT1, LMX1B, CPAMD8 ) known to cause various forms of early-onset glaucoma were initially screened for pathogenic mutations. If no plausible causative variants were identified, the exome data was examined for novel pathogenic variants. Disease-causing mutations are expected to be rare, disrupt protein function, and have high estimates of pathogenicity. Variants were filtered using 1) minor allele frequency (MAF) from the Exome Aggregation Consortium (ExAC) database (MAF less than 0.001 were retained), 2) tools to score variants according to phylogenetic conservation (GERP) and its effect on protein structure (SIFT, PolyPhen, conserved predicted pathogenic variants were obtained), 3) ocular expression, and any known functional or human disease involvement (ocular expression was prioritized) and 4) examining segregation in all available family members. The family A and B pedigree structures suggested dominant inheritance; hence, heterozygous variants were prioritized. Sanger sequencing was used to investigate segregation of candidate variants in all available family members.

\section{EFEMP1 expression vector}

To evaluate the cellular expression and localization of disease-associated EFEMP1 variants we used a pENTR/D-TOPO entry vector (Invitrogen, Carlsbad, CA) with cloned wild-type EFEMP1cDNA (provided as a gift from Dr. Rosario Fernandez-Godino, (Garland et al., 2021). The EFEMP1 mutants (p.Asn80Tyr, p.Arg140Trp, p.Arg345Trp, p.Arg477Cys) were created using the QuikChange Site-Directed Mutagenesis kit (Agilent Technologies, Santa Clara, CA). To create the p.Ter494Glnext*29 mutation, a gBlocks Gene Fragment (Integrated DNA Technologies, Iowa) was designed and cloned in to a pENTR/D-TOPO entry vector. The wild-type and mutant entry clones were verified by sequencing analysis and moved into a Gateway destination expression vector modified to contain an N-terminal V5 epitope tag in-frame (pCAG-V5-IRESEGFP) (Zhang et al., 2012) by LR recombination. Plasmid DNA was purified using the EndoFree Plasmid Maxi kit (Qiagen, Valencia, CA).

\section{Cell culture}

COS-7 cells were used to express EFEMP1 protein. The COS-7 cells were seeded in 6 -well plates at a concentration of 100,000 cells per $\mathrm{mL}$ and allowed to grow in DMEM $+10 \%$ FBS condition media. The cells were transfected with expression clones encoding wild-type or mutantEFEMP1 cDNA using Lipofectamine 3000 (Invitrogen, Carlsbad, CA) 24 hours post-seeding at 80-90\% confluence.

\section{Immunocytochemistry}

To visualize the cellular localization of EFEMP1 protein, cells were seeded on clear coverslips (Neuvitro Corp., Camas, WA). In order to visualize the endoplasmic reticulum, CellLight ER-RFP, BacMam 2.0 (Invitrogen, Carlsbad, CA) was added to each well during transfection. The cells were processed for immunocytochemistry 48 hours after transfection. Condition media was aspirated, coverslips washed with PBS, and fixed in 4\% PFA. Cells were permeabilized using 0.5\% Triton X-100 and blocked in 3\% BSA with PBS, followed by incubation in V5 Tag Monoclonal Antibody (Thermofisher, Waltham, MA) overnight. Coverslips 
were retrieved and incubated in Goat anti-Mouse IgG $(\mathrm{H}+\mathrm{L})$ Highly Cross-Adsorbed Secondary Antibody, Alexa Fluor Plus 647 (Invitrogen, Carlsbad, CA) for 2 hours and DAPI (Thermofisher, Waltham, MA) for 1 minute. Coverslips were mounted on clear slides with ProLong Glass Antifade Mountant (Invitrogen, Carlsbad, CA). Imaging of the transfected cells was done using a confocal laser scanning microscope (SP8, Leica Microsystems, Buffalo Grove, IL).

\section{Western blots}

To quantify transfected EFEMP1 protein, cells were harvested 48 hours post transfection (average transfection efficiency $75 \%$ ) (Table S1). Whole cell lysates were prepared by careful aspiration of condition media and adding cold RIPA buffer (Sigma-Aldrich, St. Louis, MO) and Pierce Protease Inhibitor (Thermofisher, Waltham, MA) to each well and agitating for 30 minutes at $4^{\circ} \mathrm{C}$. After incubation, cells were transferred to Eppendorf tubes and centrifuged for 30 minutes at $4^{\circ} \mathrm{C}$. The supernatant was retrieved and the protein concentration was determined using the Pierce BCA Protein Assay Kit (Thermofisher, Waltham, MA). For each vector group, $5 \mathrm{ug}$ of protein was mixed with $4 \mathrm{X}$ Protein Sample Loading Buffer (Li-Cor Biosciences, Nebraska) and water for a total volume of $12 \mathrm{ul}$. Each sample was denatured by heating at $95 \operatorname{deg}$ for 3 minutes and loaded in to a 15 well 4-20\% Mini-PROTEAN(r) TGX Precast Protein Gel (Bio-Rad, Hercules, CA). Electrophoresis was performed at $120 \mathrm{v}$ for approximately 1.5 hours. The gel was retrieved and transferred to a PVDF blotting membrane using the iBlot 2 system (Invitrogen, Carlsbad, CA). For normalization of protein loading, total protein staining was done using the Revert 700 Total Protein Stain Kit (Li-Cor Biosciences, Nebraska), following the manufacturer's protocol. After imaging in the 700nm channel using the Odyssey(r) Imaging System (Li-Cor Biosciences, Nebraska), the membrane was blocked for 1 hour at room temperature using Intercept(r) Blocking Buffer (Li-Cor Biosciences, Nebraska), then overnight incubation with Anti-Fibulin-3 Antibody (mab3-5): sc-33722 (Santa Cruz Biotechnology, Dallas, TX) diluted with blocking buffer to a final concentration of 1:100. After overnight incubation, the membrane was incubated in IRDye(r) 800CW secondary goat anti-mouse antibody (Li-Cor Biosciences, Nebraska), at a final concentration of 1:10,000 for 1 hour and then imaged in the $800 \mathrm{~nm}$ channel. Western blot quantification and analysis was carried out using ImageStudioLite(r) Software (Li-Cor Biosciences, Nebraska). Relative EFEMP1 abundance was calculated as the ratio between the EFEMP1 signal and the corresponding total protein signal for each sample. Three biological repeats were done for each EFEMP1 condition. Differences between mutant and wildtype EFEMP1 were tested using Student's t-test with Bonferroni correction.

\section{Statistical Analysis}

All the analysis were done using GraphPad Prism software (GraphPad Software, San Diego, CA). Adjusted p value [?] 0.05 was considered significant.

\section{RESULTS}

\section{Identification of rare coding EFEMP1 variants in three families with JOAG.}

Whole exome sequencing was completed for selected affected and unaffected family members, and exome data was filtered to retain rare variants $(\mathrm{MAF}<0.1 \%)$ that were protein altering and predicted to be pathogenic using in silico programs. Mutations were identified in known disease-causing genes in 4 families, three with MYOC mutations (p.Ter505Trpext*42; p.Gln337Arg; p.Thr438Ile) and one with a PAX6 mutation (p.Tyr296*). Novel variants of interest (Table S2) were further evaluated for co-segregation with disease in each pedigree using Sanger sequencing. Rare EFEMP1 (MIM: 601548) coding variants (GenBank: NM_001039348.3) were identified in all affected individuals of 3 of the remaining families as follows: (c.238A $>$ T, p. Asn80Tyr) in 16 affected individuals of Family A and not in 18 unaffected family members older than age 18; (c.1480T $>$ C, p. Ter494Glnext*29) was identified in 17 affected family members in family B and not in any family members older than age 18. One Family A member (age 13) and 4 Family B members (ages 7-12) were identified as mutation carriers, but are likely to be too young to manifest disease. Additionally, a third variant (c.1429C > T, p.Arg477Cys) was identified in a single case (Family C) (Figure 1A).

Patients with EFEMP1 variants ( $\mathrm{N}=34$ in total for all three families) exhibited severe disease with average 
age of disease onset of 16 years (range 3-43). Affected individuals had much higher than average IOP $(28 \mathrm{mmHg})$ and $76 \%$ were blind in at least one eye (Table 1). The majority of patients required surgical treatment for IOP elevation, however not all patients had access to clinical care. Retinal examination did not reveal evidence of subretinal deposits (drusen) characteristic of MLVT/DHRD in affected individuals (Figure 2).

The three variants p.Asn80Tyr, p.Ter494Glnext*29, and p.Arg477Cys, identified in affected individuals are not present in population databases including gnomAD and TOPMed and have not been previously reported (the stop loss variant, p.Ter494Glnext*29 is not the same variant as reported in a small Chinese adult onset glaucoma family) (Liu et al., 2020). All three variants are predicted to be deleterious by in silico programs SIFT and Polyphen2 (Table S3). p.Asn80Tyr is located within the first EGF-domain, while p.Arg477Cys and p.Ter494GLNext*29 are within the terminal fibulin-like carboxy terminal domain. p.Ter494GLNext*29 results from replacement of the wild type stop codon with a glutamine residue and the addition of 29 amino acid residues to the polypeptide before a stop codon is encountered (Figure 1B).

\section{Expression and localization of EFEMP1 wild-type and mutant proteins}

EFEMP1 is expressed in multiple ocular anterior segment cell types especially the trabecular meshwork beam cells and juxtacanilicular matrix cells (Figure 3) (van Zyl et al., 2020), two structures necessary for outflow of intraocular fluid (aqueous humor) and maintenance of normal IOP levels. EFEMP1 ocular expression is similar to Myocilin ( $M Y O C$ ) also an extracellular matrix protein involved in JOAG. MYOC mutations cause protein misfolding and endoplasmic reticulum aggregation, and efforts to reduce mutation-related misfolding can result in lower IOP in animal models (Orwig et al., 2014; Jain et al., 2017).

To assess the functional effects of the JOAG-related EFEMP1 variants, we hypothesized that these variants could cause protein misfolding, aggregation and intracellular retention similar to that observed for the p.Arg345Trp MLVT/DHRD variant and for JOAG-relatedMYOC mutations. To test this hypothesis we transfected COS7 cells with Gateway destination expression vectors (pCAG-V5-IRES-EGFP) (Zhang et al., 2012) for wildtype EFEMP1 cDNA, the three JOAG-relatedEFEMP1 variants, and also the MLVT/DHRD variant (p.Arg345Trp) and the POAG-related variant (p.Arg140Trp). Imaging transfected cells showed that wild type EFEMP1 was distributed throughout the cell, while significant intracellular aggregation was observed for each JOAG-related variant (Figure 4). Co-localization with an endoplasmic reticulum (ER) marker, CellLight ER-RFP, BacMam 2.0 (Invitrogen, Carlsbad, CA), suggests that EFEMP1 protein aggregates are formed in the vicinity of the ER. In contrast, the p.Arg345Trp variant exhibited more diffuse cellular distribution as did the POAG related variant p.Arg140Trp.

To measure intracellular protein retention, we collected an equal number of transfected cells from experiments using the wild type vector, the 3 JOAG-related vectors and the p.Arg345Trp and p.Arg140Trp vectors. Using Western blot assays (Figure 5A, Figure S1) we determined the fraction of EFEMP1 protein retained within the cell in comparison to overall protein and relative to wild type protein (Figure 5B). Compared to wild type cells intracellular EFEMP1 protein was significantly increased for all three of the JOAG-related variants $(\mathrm{P}<0.05)$. JOAG-related intracellular protein was also increased compared to the MLVT/DHRD variant p.Arg345Trp and the POAG-related p.Arg140Trp.

\section{Discussion}

Collectively, these data support the hypothesis that EFFMP1 coding variants can cause JOAG by a mechanism that involves intracellular protein aggregation and retention. Similarly, JOAG-causing MYOC mutations also promote formation of intracellular protein aggregates and the extent of protein aggregation appears to correlate with disease severity (Patterson-Orazem et al., 2019). Misfolded myocilin is hypothesized to cause ER stress leading to cellular dysfunction and potentially apoptosis (Yam et al., 2007), however the specific cells that are impacted and the underlying molecular events are not known. Studies of human and mouse MYOC knockouts (Kim et al., 2001; Pang et al., 2002; Wiggs et al., 2001) and mouse knockins (Kim et al., 2001); Zode et al., 2011) indicate that MYOC mutations are gain-of-function. The observation that the retina and optic nerves ofEFEMP1 knockout mice are anatomically normal (McLaughlin et 
al., 2007; Stanton et al., 2017; Daniel et al., 2020) suggests thatEFEMP1 loss of function also does not underlie glaucoma development. $M Y O C$ and EFEMP1 are among a group of proteins expressed in ocular extracellular matrix that also includes Thrombospondin1 (THBS1), and Angiopoietin-like 7 (ANGPTL7 ) among other proteins with potential glaucoma involvement (Wirtz et al., 2021; Tanigawa et al., 2020). Although the specific mechanisms underlying the contribution of ECM proteins to glaucoma is not known, preventing mutant $M Y O C$ expression (Jain et al., 2017) or encouraging secretion of misfolded myocilin can reduce intraocular pressure in mice (Zode et al., 2011; Zode et al., 2021), and similar approaches may also be therapeutically useful for patients withEFEMP1 mutations.

In this study we showed that the JOAG associated variants demonstrate increased intracellular protein retention compared with the MLVT/DHRD variant p.Arg345Trp. EFEMP1 p.Arg345Trp has recently been shown to effect cholesterol efflux (Tsai et al., 2021) which may potentially impact the contribution of this mutation to the characteristic extracellular protein aggregation observed in this disease (Fu et al., 2007). While some POAG genomic loci include genes that may influence cholesterol efflux (ABCA1, ARHGEF12, CAV1/2 ) (Gharahkhani et al., 2021; Springelkamp et al., 2015; Jacobo-Albavera et al., 2021; Wang et al., 2014; Okuhira et al., 2010), the role of cholesterol metabolism in glaucoma is not clear and given the involvement of EFEMP1 in JOAG could be interesting to explore further. It is also of interest that only the single missense allele p.Arg345Trp is known to cause MLVT/DHRB, yet at least 3 protein variants can cause glaucoma. It is possible thatEFEMP1 missense alleles are more likely to effect cells of the ocular outflow pathway than the retinal pigment epithelial cells involved in MVLT/DHRB. Interestingly, patients with MVLT/DHRB are not known to be at increased risk of glaucoma, and the JOAG patients withEFEMP1 mutations do not have any evidence of the MVLT/DHRB retinal dystrophy (Figure 2).

Additionally, we show that the JOAG EFEMP1 variants demonstrate increased intracellular retention compared to a possible POAG associated variant, p.Arg140Trp found in a family with 5 members affected by adult-onset POAG (Mackay et al., 2015), a finding that may be related to the less severe and genetically complex adult-onset POAG. Our results could support a contribution of p.Arg140Trp to POAG in this family, which is interestingly of African American ancestry. Exome-based studies of African American POAG cases or families have not yet been completed and further investigation in this population could reveal additionalEFEMP1 disease-related variants. A stop lost variant (Ter464Gluext*29) similar to the mutation affecting JOAG family B (Ter464Glnext*29) has recently been described in 3 members of a Chinese adult POAG family (Liu et al., 2020), While individuals in this family are described as affected by POAG, the age of disease onset is the mid-20s which would meet our diagnostic criteria for JOAG and is consistent with our results.

EFEMP1 is located on chromosome $2 \mathrm{p} 16$ within a genomic region that was initially identified as a POAG genomic locus (GLC1H) in a linkage study of a Jamaican family and European Caucasian families from the UK (Suriyapperuma et al., 2007). Subsequently linkage to this region has also been observed in four Chinese families, including two with disease onset before age 40 (Liu et al., 2012; Liu et al., 2008). POAG candidate association studies have also implicated SNPs in the 2 p16 region in cases and controls from Barbados (Jiao et al., 2009), an African-American cohort (Liu et al., 2010), Chinese (Chen et al., 2012) and South Indian cohorts (Balasubbu et al., 2021). Together with our results, these studies support EFEMP1 as the GLC1H gene and also could suggest that EFEMP1 is more commonly associated with glaucoma in non-white populations.

Our findings also suggest a phenotype-genotype spectrum that correlates the extent of intracellular protein retention with disease phenotype and extend the EFEMP1 phenotypic spectrum to include severe childhoodonset glaucoma. The mutations causing JOAG cause more severe intracellular protein retention compared to the MLVT/DHRD EFEMP1 variant and also to a variant found in an adult-onset glaucoma family. Further study will be required to determine the mechanism underlying intracellular protein retention as well as the molecular events leading to elevated IOP and glaucoma.

This study investigates the etiology of JOAG in a collection of affected families from the Philippines, a population with diverse ancestry including Asian, African and European Caucasian origins (Larena et al., 
2021). Among the pedigrees that we have collected we find thatEFEMP1 variation is a relatively common cause of childhood glaucoma (20\%), equal to $M Y O C$ in this population. Interestingly,EFEMP1 variants have not been observed in any prior studies of childhood glaucoma which have focused primarily of families with European Caucasian or Asian ancestry (Allen et al., 2015; Huang et al., 2018). As well, MYOC and other currently known childhood gene mutations have infrequently been identified in patients with African ancestry (Liu et al., 2012). These results suggest that investigation of diverse populations such as this Filipino cohort will be necessary to develop a more comprehensive set of childhood glaucoma genes.

The discovery of genes causing childhood glaucoma makes it possible to use genetic testing to inform genetic counseling for affected families. Treatment initiated at early stages of disease can delay irreversible optic nerve degeneration and provide the best chance that an affected child will maintain useful sight throughout their lifetime. Informed genetic counseling makes it possible to create surveillance and treatment plans for mutation carriers and alleviates the burden of screening family members without disease-causing mutations. Currently however a molecular diagnosis can only be achieved for approximately 20-25\% of cases based on known genes (Allen et al., 2015). Discovery of novel disease-causing genes such as EFEMP1 is needed to improve the overall diagnostic yield and effectiveness of childhood glaucoma genetic testing.

In conclusion we have identified 3 different EFEMP1 coding variants that segregate with a severe form of glaucoma affecting children in 3 independent families from the Philippines. Our results suggest that diseaseassociated variants cause significant intracellular EFEMP1 aggregation and retention and that the extent of intracellular retention appears to be correlated with EFEMP1-related disease phenotypes. This study further supports a role for EFEMP1 in ocular extracellular matrix and in regulation of intraocular fluid dynamics and IOP and provides new opportunities for genetic testing and therapeutic intervention.

\section{Data availability statement}

The authors confirm that the data supporting the findings of this study are available within the article or supplementary information. All variants identified in the present study have been submitted to ClinVar (https://www.ncbi.nih.gov/clinvar/) and are awaiting accession numbers.

\section{Acknowledgments}

Supported by the ARVO Foundation David L. Epstein award (ERAC) and NIH/NEI R01 EY031830 ad P30EY014104 (JLW)

\section{Declaration of Interests}

JLW has received research support from Aerpio pharmaceuticals and is a consultant for Aerpio, Allergan, Maze, Editas, Regenxbio and Avellino.

\section{Web Resources}

gnomAD, https://gnomad.broadinstitute.org/

TOPMed, https://bravo.sph.umich.edu/freeze8/hg38/

SIFT, https://sift.bii.a-star.edu.sg/

Polyphen2, http://genetics.bwh.harvard.edu/pph2/

Genbank, https://www.ncbi.nlm.nih.gov/genbank/

OMIM, https://www.omim.org/

\section{References}

Allen KF, Gaier ED, Wiggs JL. (2015) Genetics of Primary Inherited Disorders of the Optic Nerve: Clinical Applications. Cold Spring Harb Perspect Med. 5, a017277. 
Balasubbu S, Krishnadas SR, Jiao X, Hejtmancik JF, Sundaresan P (2012) Evaluation of SNPs on chromosome $2 p$ with primary open angle glaucoma in the South Indian cohort. Invest Ophthalmol Vis Sci 53: 1861-1864.

Chen LJ, Tam PO, Leung DY, Fan AH, Zhang M, Tham CC, et al. (2012) SNP rs1533428 at 2p16.3 as a marker for late-onset primary open-angle glaucoma. Mol Vis 18: 1629-1639.

Consugar MB, Navarro-Gomez D, Place EM, Bujakowska KM, Sousa ME, Fonseca-Kelly ZD, Taub DG, Janessian M, Wang DY, Au ED, Sims KB, Sweetser DA, Fulton AB, Liu Q, Wiggs JL, Gai X, Pierce EA (2015). Panel-based genetic diagnostic testing for inherited eye diseases is highly accurate and reproducible, and more sensitive for variant detection, than exome sequencing. Genet Med. 17:253-261.

Daniel S., Renwick M., Chau V.Q., Datta S., Maddineni P., Zode G., Wade E.M., Robertson S.P., Petroll W.M., Hulleman J.D. (2020) Fibulin-3 knockout mice demonstrate corneal dysfunction but maintain normal retinal integrity. J Mol Med (Berl). 98, 1639-1656.

Fu L., Garland D., Yang Z., Shukla D., Rajendran A., Pearson E., Stone E.M., Zhang K., Pierce E.A. (2007) The R345W mutation in EFEMP1 is pathogenic and causes AMD-like deposits in mice. Hum. Mol. Genet. $16,2411-2422$.

Garland DL, Pierce EA, Fernandez-Godino R (2021). Complement C5 is not critical for the formation of sub-RPE deposits in Efemp1 mutant mice. Sci Rep. 11:10416.

Gharahkhani P., Jorgenson E., Hysi P, Khawaja A.P., Pendergrass S., Han X., Ong J.S., Hewitt A.W., Segre A.V., Rouhana J.M., et al. (2021) Genome-wide meta-analysis identifies 127 open-angle glaucoma loci with consistent effect across ancestries. Nat. Commun. 12, 1258.

Huang C, Xie L, Wu Z, Cao Y, Zheng Y, Pang CP, Zhang M. (2018) Detection of mutations in MYOC, OPTN, NTF4, WDR36 and CYP1B1 in Chinese juvenile onset open-angle glaucoma using exome sequencing. Sci Rep. 8, 4498.

Jacobo-Albavera L, Dominguez-Perez M, Medina-Leyte DJ, Gonzalez-Garrido A, Villarreal-Molina T. (2021) The Role of the ATP-Binding Cassette A1 (ABCA1) in Human Disease. Int J Mol Sci. 22, 1593.

Jain A., Zode G., Kasetti R.B., Ran F.A., Yan W., Sharma T.P., Bugge K., Searby C.C., Fingert J.H., Zhang F., et al. (2017) CRISPR-Cas9-based treatment of myocilin-associated glaucoma. Proc. Natl. Acad. Sci. U. S. A. 2017 Oct 17;114(42):11199-11204.

Jiao X, Yang Z, Yang X, Chen Y, Tong Z, Zhao C, et al. (2009) Common variants on chromosome 2 and risk of primary open-angle glaucoma in the Afro-Caribbean population of Barbados. Proc Natl Acad Sci U S A 106: 17105-17110.

Khawaja A.P., Cooke Bailey J.N., Wareham N.J., Scott R.A., Simcoe M., Igo R.P. Jr, Song Y.E., Wojciechowski R., Cheng C.Y., Khaw P.T., et al. (2018) Genome-wide analyses identify 68 new loci associated with intraocular pressure and improve risk prediction for primary open-angle glaucoma. Nat. Genet. 50, 778-782.

Kim B.S., Savinova,O.V., Reedy M.V., Martin J., Lun Y., Gan L., Smith R.S., Tomarev S.I., John S.W., Johnson R.L. (2001) Targeted Disruption of the Myocilin Gene (Myoc) Suggests that Human GlaucomaCausing Mutations Are Gain of Function Mol Cell Biol 21, 7707-7713.

Kobayashi N., Kostka G., Garbe J.H.O., Keene D.R., Bachinger H.P., Hanisch F.-G., Markova D., Tsuda T., Timpl R., Chu M.-L., Sasaki T. (2007) A comparative analysis of the fibulin protein family. Biochemical characterization, binding interactions, and tissue localization. J. Biol. Chem. 282, 11805-11816.

Larena M., Sanchez-Quinto F., Sjodin P., McKennaJ., Ebeo C., Reyes R. Casel O., Huang J.Y., Hagada K.P., Guilay D. (2021). Multiple migrations to the Philippines during the last 50,000 years. Proc. Natl. Acad. Sci. U S A 118, e2026132118. 
Lin Y, Liu T, Li J, Yang J, Du Q, Wang J, et al. (2008) A genome-wide scan maps a novel autosomal dominant juvenile-onset open-angle glaucoma locus to 2p15-16. Mol Vis 14: 739-744.

Liu T, Xie L, Ye J, Liu Y, He X (2012) Screening of candidate genes for primary open angle glaucoma. Mol Vis 18: $2119-2126$.

Liu T., Tang C., Shi X. (2020) Analysis of variants in Chinese individuals with primary open-angle glaucoma using molecular inversion probe (MIP)-based panel sequencing. Mol. Vis. 26, 378-391.

Liu W, Liu Y, Challa P, Herndon LW, Wiggs JL, Girkin CA, Allingham RR, Hauser MA. (2012) Low prevalence of myocilin mutations in an African American population with primary open-angle glaucoma. Mol. Vis. 18, 2241-2246.

Liu Y, Qin X, Schmidt S, Allingham RR, Hauser MA (2010) Association between chromosome 2p16.3 variants and glaucoma in populations of African descent. Proc Natl Acad Sci U S A 107: E61; author reply E62.

Mackay D.S., Bennett T.M., Shiels A. (2015) Exome Sequencing Identifies a Missense Variant in EFEMP1 Co-Segregating in a Family with Autosomal Dominant Primary Open-Angle Glaucoma. PLoS One. 10, e0132529.

Marmorstein L.Y., Munier F.L., Arsenijevic Y., Schorderet D.F., McLaughlin P.J., Chung D., Traboulsi E., Marmorstein A.D. (2002) Aberrant accumulation of EFEMP1 underlies drusen formation in Malattia Leventinese and age-related macular degeneration. Proc. Natl. Acad. Sci. U. S. A. 99, 13067-13072.

McLaughlin P.J., Bakall B., Choi J., Liu Z., Sasaki T., Davis E.C., Marmorstein A.D., Marmorstein L.Y. (2007) Lack of fibulin-3 causes early aging and herniation, but not macular degeneration in mice. Hum. Mol. Genet. 16, 3059-3070.

Okuhira K., Fitzgerald M.L., Tamehiro N., Ohoka N., Suzuki K., Sawada J.-I., Naito M., Nishimaki-Mogami T. (2010) Binding of PDZ-RhoGEF to ATP-binding cassette transporter A1 (ABCA1) induces cholesterol efflux through RhoA activation and prevention of transporter degradation J. Biol. Chem. 285, 16369-16377.

Orwig S.D., Chi P.V., Du Y., Hill S.E., Cavitt M.A., Suntharalingam A., Turnage K.C., Dickey C.A., France S., Fu H., Lieberman R.L. (2014) Ligands for glaucoma-associated myocilin discovered by a generic binding assay. ACS Chem. Biol. 9, 517-525.

Pang C.P., et al. (2002) TIGR/MYOC gene sequence alterations in individuals with and without primary open-angle glaucoma. Invest. Ophthalmol. Vis. Sci. 43, 3231-3235.

Papadopoulos M., Vanner E.A., Grajewski A.L., International Study of Childhood Glaucoma - Childhood Glaucoma Research Network Study Group. (2020) International Study of Childhood Glaucoma. Ophthalmol. Glaucoma. 3(2), 145-157.

Patterson-Orazem A.C., Hill S.E., Wang Y., Dominic I.M., Hall C.K., Lieberman R.L. (2019) Differential Misfolding Properties of Glaucoma-Associated Olfactomedin Domains from Humans and Mice. Biochemistry. $58,1718-1727$.

Springelkamp H, Iglesias AI, Cuellar-Partida G, Amin N, Burdon KP, van Leeuwen EM, Gharahkhani P, Mishra A, van der Lee SJ, Hewitt AW, et al. (2015) ARHGEF12 influences the risk of glaucoma by increasing intraocular pressure. Hum Mol Genet. 24, 2689-2699.

Springelkamp H, Mishra A, Hysi PG, Gharahkhani P, Hohn R, Khor CC, Cooke Bailey JN, Luo X, Ramdas WD, Vithana E, et al. (2015) Meta-analysis of Genome-Wide Association Studies Identifies Novel Loci Associated With Optic Disc Morphology. Genet. Epidemiol. 39, 207-216.

Stanton J.B., Marmorstein A.D., Zhang Y., Marmorstein L.Y. (2017) Deletion of Efemp1 Is Protective Against the Development of Sub-RPE Deposits in Mouse Eyes. Invest Ophthalmol Vis Sci. 58, 1455-1461. 
Stone EM, Lotery AJ, Munier FL, Heon E, Piguet B, Guymer RH, Vandenburgh K, Cousin P, Nishimura D, Swiderski RE, et al. (1999) A single EFEMP1 mutation associated with both Malattia Leventinese and Doyne honeycomb retinal dystrophy. Nat. Genet. 22, 199-202.

Suriyapperuma SP, Child A, Desai T, Brice G, Kerr A, Crick RP, et al. (2007) A new locus (GLC1H) for adult-onset primary open-angle glaucoma maps to the 2p15-p16 region. Arch Ophthalmol 125: 86-92.

Tanigawa Y., Wainberg M., Karjalainen J., Kiiskinen T., Venkataraman G., Lemmela S., Turunen J.A., Graham R.R., et al. (2020) Rare protein-altering variants in ANGPTL7 lower intraocular pressure and protect against glaucoma. PLoS Genet. 16, e1008682.

Tsai Y.T., Li Y., Ryu J., Su P.Y., Cheng C.H., Wu W.H., Li Y.S., Quinn P.M.J., Leong K.W., Tsang S.H. (2021) Impaired cholesterol efflux in retinal pigment epithelium of individuals with juvenile macular degeneration. Am. J. Hum. Genet. 108, 903-918.

Vaclavik V., Tran H.V., Schorderet D.F. (2020) Malattia Leventinese: EFEMP1 R345W Variant Is a Hot Spot Mutation, Not a Founder Mutation. Ophthalmol Retina. 4, 1023.

van Zyl T., Yan W., McAdams A., Peng Y-R., Shekhar K., Regev A., Juric D., Sanes J.R. (2020) Cell atlas of aqueous humor outflow pathways in eyes of humans and four model species provides insight into glaucoma pathogenesis. Proc Natl Acad Sci U S A 117, 10339-10349.

Wagner AH, Anand VN, Wang WH, Chatterton JE, Sun D, Shepard AR, Jacobson N, Pang IH, Deluca AP, Casavant TL, Scheetz TE, Mullins RF, Braun TA, Clark AF (2013) Exon-level expression profiling of ocular tissues. Exp Eye Res. 111:105-111.

Wang F, Gu HM, Zhang DW. (2014) Caveolin-1 and ATP binding cassette transporter A1 and G1-mediated cholesterol efflux. Cardiovasc Hematol Disord Drug Targets. 2014;14(2):142-8.

Wang, K., Gaitsch, H., Poon, H., Cox, N.J., Rzhetsky, A. (2017) Classification of common human diseases derived from shared genetic and environmental determinants. Nat. Genet. 49, 1319-1325.

Weinreb R. N., Aung T., Medeiros F. A. (2014) The pathophysiology and treatment of glaucoma: A review. JAMA 311, 1901-1911.

Weinreb R.N., Leung C.K., Crowston J.G., Medeiros F.A., Friedman D.S., Wiggs J.L., Martin K.R. (2016) Primary open-angle glaucoma. Nat. Rev. Dis. Primers. 2, 16067.

Wiggs J.L., Pasquale L.R. (2017) Genetics of glaucoma. Hum. Mol. Genet. 26, R21-R27.

Wiggs J.L., Vollrath D. (2001) Molecular and clinical evaluation of a patient hemizygous for TIGR/MYOC. Arch. Ophthalmol. 119, 1674-1678.

Wirtz M.K., Sykes R., Samples J., Edmunds B., Choi D., Keene D.R., Tufa S.F., Sun Y.Y., Keller K.E. (2021) Identification of missense extracellular matrix gene variants in a large glaucoma pedigree and investigation of the N700S thrombospondin-1 variant in normal and glaucomatous trabecular meshwork cells. Curr. Eye. Res. 2021 Jun 18

Yam G.H., Gaplovska-Kysela K., Zuber C., Roth J. (2007) Aggregated myocilin induces Russell bodies and causes apoptosis: Implications for the pathogenesis of myocilin-caused primary open-angle glaucoma. Am. J. Pathol. 170, 100-109.

Zhang Q., Liu Q., Austin C., Drummond I., Pierce E.A. (2012). Knockdown of ttc26 disrupts ciliogenesis of the photoreceptor cells and the pronephros in zebrafish. Mol. Biol. Cell. 23,3069-3078.

Zode GS, Bugge KE, Mohan K, Grozdanic SD, Peters JC, Koehn DR, Anderson MG, Kardon RH, Stone EM, Sheffield VC. Topical ocular sodium 4-phenylbutyrate rescues glaucoma in a myocilin mouse model of primary open-angle glaucoma. Invest Ophthalmol Vis Sci. 2012 Mar 21;53(3):1557-65. 
Zode GS, Kuehn MH, Nishimura DY, Searby CC, Mohan K, Grozdanic SD, Bugge K, Anderson MG, Clark AF, Stone EM, Sheffield VC. Reduction of ER stress via a chemical chaperone prevents disease phenotypes in a mouse model of primary open angle glaucoma. J Clin Invest. 2011 Sep;121(9):3542-3553.

\begin{tabular}{|c|c|c|}
\hline Family-pedigree position & Sex & Age diagnosed w/gl \\
\hline A-I:2 & $\mathrm{F}$ & 11 \\
\hline A-II:1 & M & 17 \\
\hline A-II:4 & $\mathrm{F}$ & 12 \\
\hline A-II:8 & $\mathrm{F}$ & 28 \\
\hline A-II:10 & $\mathrm{F}$ & 11 \\
\hline A-III:1 & $\mathrm{F}$ & 22 \\
\hline A-III:8 & $\mathrm{F}$ & 10 \\
\hline A-III:9 & M & 31 \\
\hline A-III:12 & $\mathrm{F}$ & 17 \\
\hline A-III:15 & M & 18 \\
\hline A-III:29 & $\mathrm{F}$ & 28 \\
\hline A-III:32 & M & 15 \\
\hline A-III:33 & $\mathrm{F}$ & 25 \\
\hline A-III:34 & M & 18 \\
\hline A-IV:1 & M & 18 \\
\hline A-IV:16 & M & 8 \\
\hline B-III:17 & M & 19 \\
\hline B-IV:3 & M & 20 \\
\hline B-IV:8 & M & 9 \\
\hline B-IV:10 & $\mathrm{F}$ & 43 \\
\hline B-IV:18 & M & 15 \\
\hline B-IV:21 & M & 7 \\
\hline B-IV:24 & M & 7 \\
\hline B-IV:28 & $\mathrm{F}$ & 8 \\
\hline B-IV:38 & M & 18 \\
\hline $\mathrm{B}-\mathrm{V}: 1$ & $\mathrm{~F}$ & 19 \\
\hline $\mathrm{B}-\mathrm{V}: 4$ & M & 9 \\
\hline$B-V: 5$ & M & 10 \\
\hline $\mathrm{B}-\mathrm{V}: 6$ & $\mathrm{M}$ & 9 \\
\hline $\mathrm{B}-\mathrm{V}: 24$ & $\mathrm{~F}$ & 12 \\
\hline $\mathrm{B}-\mathrm{V}: 26$ & $\mathrm{~F}$ & 11 \\
\hline $\mathrm{B}-\mathrm{V}: 32$ & M & 3 \\
\hline $\mathrm{B}-\mathrm{V}: 35$ & $\mathrm{~F}$ & 11 \\
\hline C-II:1 & $\mathrm{F}$ & 18 \\
\hline Mutation carrier, no clinical signs of glaucoma & Mutation carrier, no clinical signs of glaucoma & Mutation carrier, no cli \\
\hline A-IV:11 & $\mathrm{F}$ & na \\
\hline $\mathrm{B}-\mathrm{V}: 9$ & M & na \\
\hline $\mathrm{B}-\mathrm{V}: 10$ & M & na \\
\hline B-V:11 & $\mathrm{M}$ & na \\
\hline
\end{tabular}

Table 1. Clinical findings in subjects with EFEMP1 rare coding variants. All clinical data listed were obtained during study recruitment. Normal IOP is less than 21 and visual acuities of 20/200 or greater meet the definition for blindness. NLP, LP, CF, and HM also meet the definition for blindness. Abbreviations: Single dash (-): exam not performed because of poor view, corneal opacification, or phthisis 
bulbi; na: not applicable; PAS: peripheral anterior synechiae; NLP: no light perception; LP: light perception; $\mathrm{CF}$ : counting fingers; HM: hand movement; M: male; F: female; OD: right eye; OS: left eye.

Figure titles and legends

Figure 1A. Family pedigrees with juvenile-onset open-angle glaucoma (JOAG) and EFEMP1 mutation status. Arrow indicates the proband. Squares indicate male subjects; and circles refer to female subjects. Slash through indicates a deceased individual. Solid symbols indicate subjects diagnosed with JOAG; half-filled symbols indicate subjects who carry the EFEMP1 variant but with no clinical signs of glaucoma; and open symbols refer to subjects with no glaucoma and noEFEMP1 variant. Genotypes are heterozygous mutant $(\mathrm{m} /+)$ and wild type $(+/+)$. Figure 1B. Schematic diagram of $\boldsymbol{E F E M P 1}$ and evolutionary conservation. EFEMP1 Genbank transcript NM_001039348.3 and color-coded exons corresponding to the protein domain. The mutations p.Asn80Tyr, p.Arg477Cys, and p.Ter494Glnext*29 are located at highly conserved domains across several organisms.

Figure 2. Fundus images from a patient from Family B with EFEMP1 variant p.Ter494Glnext*29 (B-V:5). The image show damage to the optic nerve but no evidence of subretinal deposits (drusen) characteristic of Malattia Leventinese/Doyne Honeycomb dystrophy.

Figure 3. Single cell RNA sequencing EFEMP1 expression and known early onset glaucoma genes from the human aqueous humor outflow pathway. Comparative expression levels of EFEMP1 and genes known to cause various types of early onset glaucoma. Plot generated from the Broad Institute of MIT and Harvard's Single Cell Portal using data from "Cell atlas of aqueous humor outflow pathways in eyes of humans and four model species provides insight into glaucoma pathogenesis" by van Zyl et al, 2019. ${ }^{16}$

Figure 4 . Expression of EFEMP1 in COS-7 cells.Cultured COS-7 cells transfected with wildtype and variant EFEMP1 and processed with immunocytochemistry and the following stains: DAPI (nucleus), CellLight ER-RFP (endoplasmic reticulum), Alexa 647 (EFEMP1 protein), GFP (GFP expression reporter). Imaged using Leica SP8 confocal microscope using 63x objective (glycerol immersion), digital zoom 2x.

Figure 5. EFEMP1 expression in COS7 cells.

A. Western blot of wild-type (WT) and mutant EFEMP1 proteins. Transfected COS7 cell-lysates showing expression of WT and mutant EFEMP1. Control group consist of cell-lysates from non-transfected COS7 cells. B. Ratio of intracellular EFEMP1 protein to total protein for wild type and each variant. Relative EFEMP1 abundance was calculated as the ratio between the EFEMP1 signal and the corresponding total protein signal in each sample measured using Revert 700 total protein stain. Three biological repeats were done for each EFEMP1 condition. Differences between mutant EFEMP1 and wildtype EFEMP1 were tested using Student's t-test with Bonferroni correction. Error bars indicate the standard error. ${ }^{*} \mathrm{p}<0.05$, $* * \mathrm{p}<0.001$ 


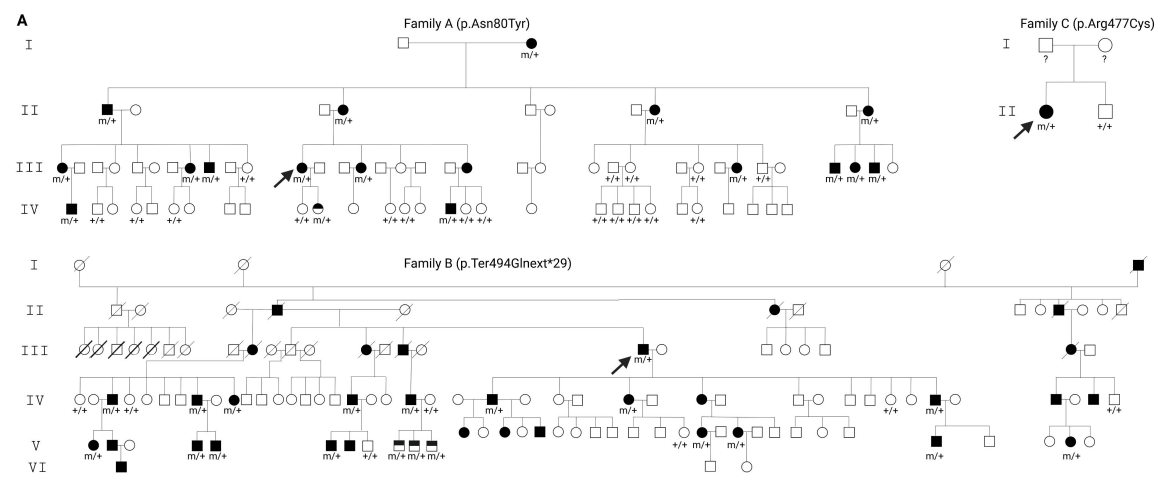

NM_ 001039348.3: EGF containing fibulin extracellular matrix protein 1

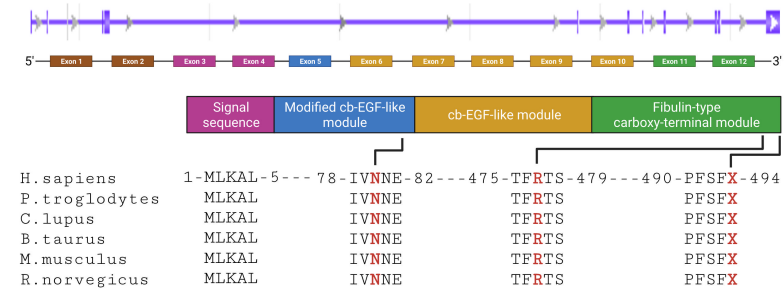
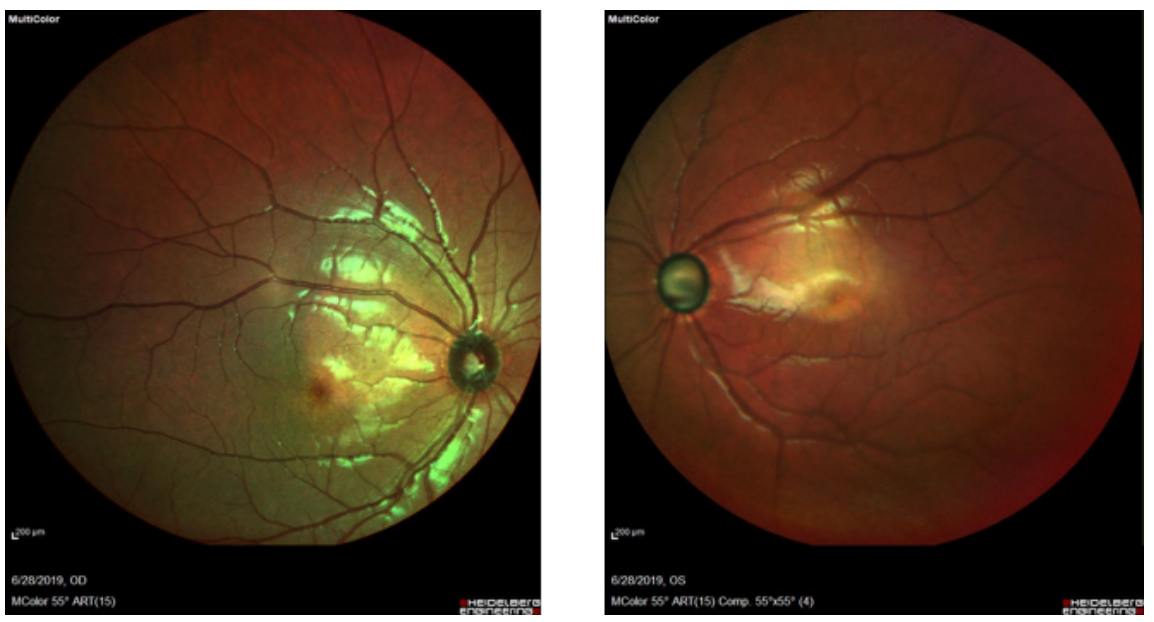


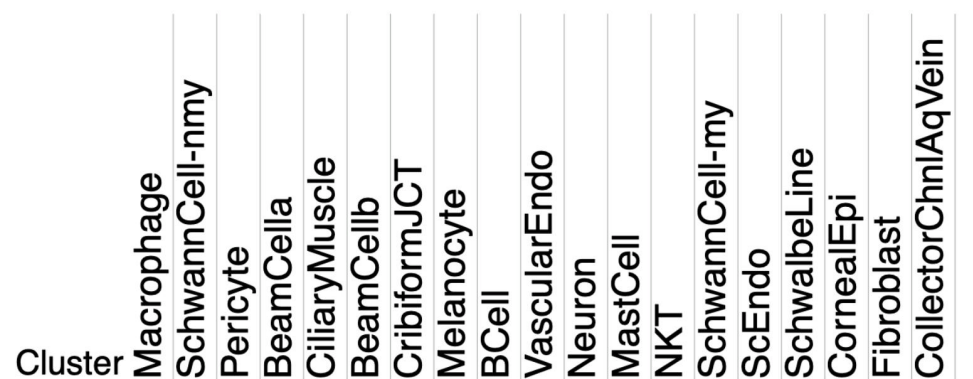

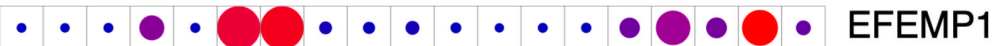

000000000000 MYOC

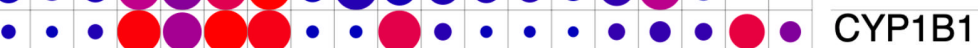

• • • •

-

- $\bullet$ O

• • • • • • • • • • • • • • • • PAX6

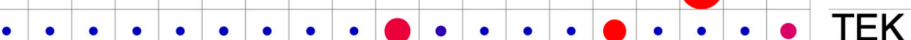

• • • • • • • • • • • • • • • •

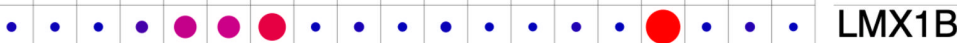

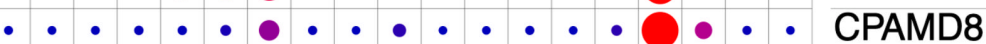

Legend
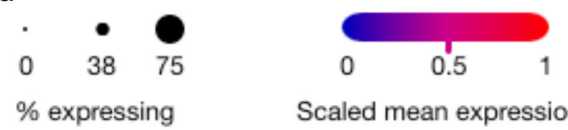

$\%$ expressing Scaled mean expression

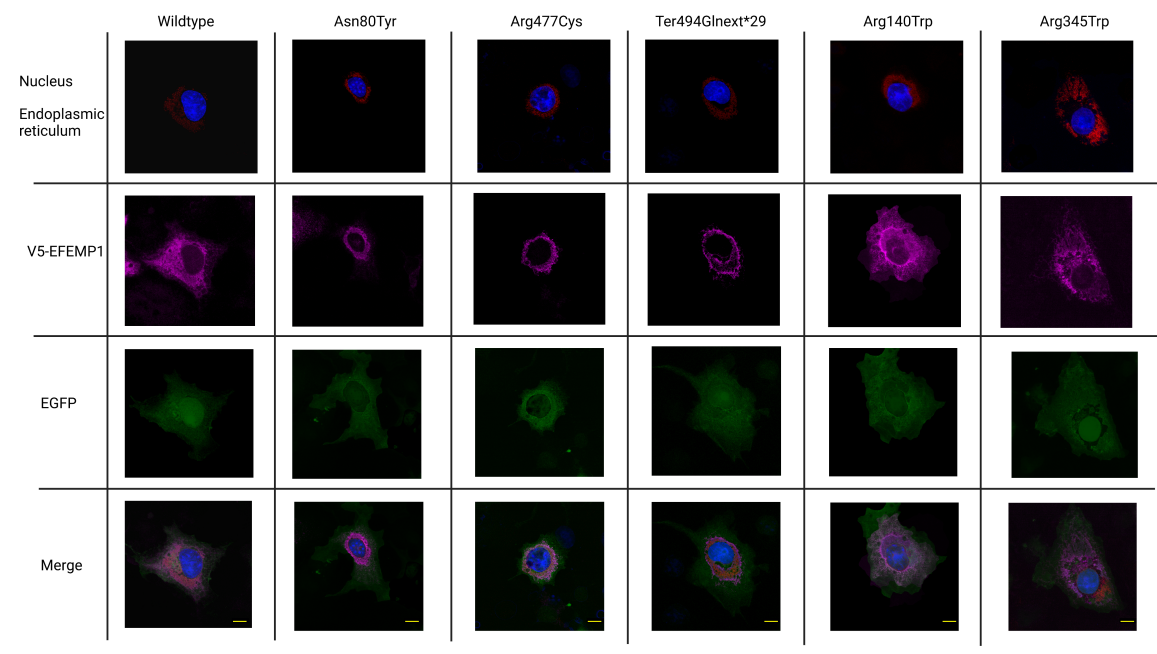




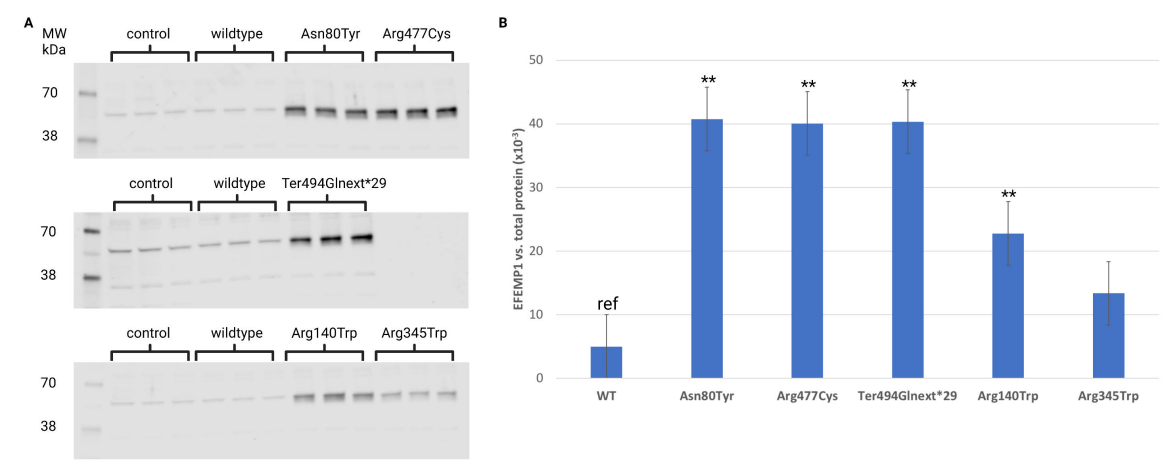

